Fifth Meeting, 10th March 1905.

Mr W. L. Thomson, President, in the Chair.

\title{
Some Proofs of Newton's Theorem on Sums of Powers of Roots.
}

By R. F. Muirhead, M.A., D.Sc.

This well-known theorem, published in Newton's Arithmetica Universalis, may be formulized thus :-

$$
s_{n}-p_{1} s_{m-1}+p_{2} s_{m-2} \ldots \mp p_{m-1} s_{1} \pm m p_{m}=0
$$

where $s_{m} \equiv$ the sum of the $m$ th powers of the roots of the equation

$$
x^{n}-p_{1} x^{n-1}+p_{2} x^{n-2} \ldots \pm p_{n}=0
$$

Of course, when $m>n$, certain coefficients $p_{n+1}, p_{n+9}, \ldots p_{n}$ will be zero, and the last term that does not vanish will be $\pm p_{n} 8_{m-n}$.

Several elementary proofs of this theorem which have not hitherto been printed have accumulated in my notes during past years. They are elementary in the sense that they do not involve the use of infinite series. The present communication has been suggested to me by a perusal of the note by $\mathrm{Mr}$ Tweedie in last session's Proceedings, where a brief and suggestive proof of the theorem is given.

Of the proofs given in the present paper, the first is one for which I am indebted to Mr John Dougall, who showed it to me several years ago. The second, perhaps, is the most direct. The third is a modification of the line of proof given in Chapter XVIII. of Chrystal's Algebra.

$$
\text { First Proof. }
$$

Denote by $F(n)$ the expression

$$
\left(s_{m}-p_{1} s_{m-1}+p_{2} s_{m-2}-\ldots \pm p_{n-1} s_{1} \mp m p_{m}\right)_{n}
$$

where $s_{r} \equiv$ the sum of the $r$ th powers of the $n$ letters $\alpha_{1}, a_{2}, \ldots a_{n}$ and $p_{r} \equiv, ", "$, products of $a_{1}, a_{2}, \ldots a_{n}$ taken $r$ at a time. Then for the case of $n=m$, the quantities $a_{1}, a_{2}, \ldots$ are the roots of the $m$-ic

$$
x^{m}-p_{1} x^{m-1}+p_{2} x^{m-2}-\ldots \pm p_{m-1} x \mp p_{m}=0 . \text {. }
$$


Substituting successively $a_{1}, a_{2}, \ldots a_{m}$ for $x$ in (3) and adding the results, we get

$$
\mathrm{F}(m)=\mathbf{0}
$$

Now suppose $n=m+1$, then if any one of the quantities $a_{1}, a_{2}, \ldots a_{n}$ vanish, $F(m+1)$ will reduce to $F(m)$ and will therefore vanish. Hence that particular a must be a factor of $F(m+1)$. Similarly we see that each of the other letters $\alpha$ must be a factor of $\mathbf{F}(m+1)$. This is only possible if $\mathrm{F}(m+1)$ is identically zero, since it is only of dimensions $m$ in the $\alpha$ 's.

$$
\text { Thus } \mathbf{F}(m+1)=0 \text { identically. }
$$

In a similar manner we can show that $\mathbf{F}(m+2)$ is identically zero, since it reduces to $F(m+1)$, and therefore to zero when one of its $m+2 a$ 's vanishes. And so on, by mathematical induction.

Thus when $n \nless m$ we have $\mathrm{F}(n)=0$.

The proof when $n<m$ is easy, by taking an $n-i c$ and multiplying by $x^{m-n}$ and then substituting $a_{1}, a_{2}, \ldots a_{n}$ successively for $x$ in it, and adding: but here, of course, $p_{n+1}, p_{n+2}, \ldots p_{m}$ are all zero, and $F(n)$ reduces to

$$
s_{m}-p_{1} s_{m-1}+p_{2} s_{m-2} \ldots \pm p_{n} s_{m-n} .
$$

\section{Second Proof.}

Given $n$ letters $a, \beta, \gamma, \ldots$ we have

(5) $\ldots \Sigma \alpha^{p} \Sigma(\alpha \beta \gamma \ldots$ to $q$ factors $)=\Sigma\left(\alpha^{p+1} \beta \gamma \ldots\right)+\Sigma\left(\alpha^{p} \beta \gamma \ldots\right.$ to $\overline{q+1}$ factors $)$

excepting when $p=1$, in which case we have

(6)... $\Sigma \alpha \Sigma(\alpha \beta \gamma \ldots$ to $q$ factors $)=\Sigma\left(\alpha^{2} \beta \gamma ..\right)+(q+1) \Sigma(\alpha \beta$. to $\overline{q+1}$ factors $)$.

Hence we deduce

$$
\begin{aligned}
& \Sigma a^{m}-\Sigma \alpha \Sigma a^{m-1}+\Sigma \alpha \beta \Sigma a^{m-2}-\ldots \pm \Sigma(\alpha \beta \gamma \ldots \text { to } m-1 \text { factors }) \Sigma a \\
& =\Sigma a^{m}-\left(\Sigma a^{m}+\Sigma a^{m-1} \beta\right)+\left(\Sigma a^{m-1} \beta+\Sigma a^{m-2} \beta \gamma\right)-\ldots \\
& \quad \mp\left\{\Sigma\left(\alpha^{3} \beta \gamma \ldots\right)+\Sigma\left(a^{2} \beta \gamma \ldots\right)\right\} \\
& \quad \pm\left\{\Sigma\left(\alpha^{2} \beta \gamma \ldots\right)+m \Sigma(\alpha \beta \gamma \ldots \text { to } m \text { factors })\right\} \\
& = \pm m \Sigma(\alpha \beta \ldots \text { to } m \text { factors }) .
\end{aligned}
$$


Thus, using the previous notation, we have

$$
s_{m}-p_{1} s_{m-1}+p_{2} s_{m-2} \ldots \pm p_{m-1} s_{1} \mp m p_{m}=0
$$

which is Newton's Theorem, excepting that $p_{1}, p_{2}, \ldots$ are here defined as symmetric functions, instead of as coefficients of an equation.

This proof applies equally for all positive integral values of $m$; but, of course, if $m>n$, the values of $p_{n+1}, p_{n+2}, \ldots p_{m}$ will be zero.

\section{Third Proof.}

Let $p_{r}$ denote the sum of the products of $n$ letters $\alpha, \beta, \gamma, \ldots$ taken $r$ at a time, and $a_{r}$ denote the same function of the $n-1$ letters $\beta, \gamma, \delta, \ldots$, and $b_{r}$ that of the $n-1$ letters $a, \gamma, \delta, \ldots$, and so on. Then it is obvious from first principles that

$$
\begin{aligned}
& p_{r}=a a_{r-1}+a_{r} \\
& p_{r-1}=a a_{r-2}+a_{r-1} \\
& \cdots \cdots \cdots \cdots \cdots \cdots \\
& p_{2}=a a_{1}+a_{2} \\
& p_{1}=\alpha \quad+a_{1} .
\end{aligned}
$$

Multiplying these equations respectively by

$$
1,-a, a^{2},-a^{3}, \ldots \pm a^{r-2}, \mp a^{r-3},
$$

and adding, we get

$$
p_{r}-a p_{r-1}+a^{2} p_{r-2} \ldots \ldots \mp a^{r-1} p_{1}=a_{r} \mp a^{r}-
$$

Similarly we can show that

$$
\begin{aligned}
& p_{r}-\beta p_{r-1}+\beta^{2} p_{r-2} \ldots \ldots \mp \beta^{r-1} p_{1}=b_{r} \mp \beta^{r} \\
& p_{r}-\gamma p_{r-1}+\gamma^{2} p_{r-2} \ldots \ldots \mp \gamma^{r-1} p_{1}=c_{r} \mp \gamma^{r}
\end{aligned}
$$

Summing these identities, we get

$$
n p_{r}-s_{1} p_{r-1}+s_{2} p_{r-2} \ldots \ldots \mp s_{r-1} p_{1}=\overline{n-r} p_{r} \mp s_{r},
$$

the factor $n-r$ being due to the fact that each term of $p_{r}$ occurs in $n-r$ of the quantities $a_{r}, b_{r}$, etc., viz., in those which do not exclude any of the $r$ letters of which that term is formed. 
Hence, transposing, we have

$$
r p_{r}-s_{1} p_{r-1}+s_{2} p_{r-2}-\ldots \ldots \mp s_{r-1} p_{1} \pm s_{r}=0 .
$$

Thus Newton's Theorem is proved for all positive integral values of $r$. Of course when $r>n$, certain $p$ 's will be zero.

This method of proof is in essential respects analogous to that given in Chrystal's Algebra, Chapter XVIII., from which, however, it differs principally with respect to its starting point, i.e., in starting from the elementary symmetric functions instead of from an equation. It is perhaps worthy of note that, as put here, the investigation enables us to deduce as a corollary the fact that $-p_{1}, p_{2},-p_{3}$, etc., are the coefficients of the $n$-ic whose roots are $\alpha, \beta, \gamma, \ldots$.

The equation (7) may be written

$$
a^{r}-p_{1} a^{r-1}+p_{2} a^{r-2} \ldots \ldots \pm p_{r}= \pm a_{r} .
$$

Putting $r=n$ and observing that in this case $a_{r}=a_{n}=0$,

we get $\quad a^{n}-p_{1} a^{n-1}+p_{2} a^{n-2} \ldots \ldots \pm p_{n}=0$.

Thus the equation $x^{n}-p_{1} x^{n-1} \ldots \pm p_{n}=0$ is satisfied by each of the $n$ quantities $a, \beta, \gamma, \ldots$; i.e., it is the equation of which these are the roots.

It may be of interest to generalise Newton's Formula to a certain extent, by using the method of the second proof.

The equation (5) may be seen to hold good for all values of $p$, whether positive, negative, or even fractional, provided we agree that in each $\Sigma$ on the right hand side of (5) one letter is to have an index attached, which may be 0 or 1 in certain cases, and that $\Sigma \alpha^{1}(\beta \gamma \delta \ldots$ to $q$ factors $)$ is the same as $(q+1) \Sigma(\alpha \beta \ldots$ to $q+1$ factors $)$ and that $\Sigma \alpha^{0}(\beta \gamma \delta \ldots$ to $q$ factors) is the same as $(n-q) \Sigma(\alpha \beta \gamma \ldots$ to $q$ factors) so that $\mathrm{S}^{0}$ is the same as $n$. The exceptional case of $p=1$

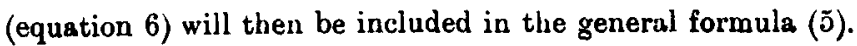


To illustrate the moditied notation, let us take the case of four quantities $a, \beta, \gamma, \delta$. Here we have

$$
\begin{aligned}
& \grave{\nu}^{1} \beta \gamma \equiv \alpha^{1} \beta \gamma+\alpha^{1} \beta \delta+\alpha^{1} \gamma \delta+\beta^{1} \alpha \gamma+\beta^{1} \alpha \delta+\beta^{1} \gamma \delta+\gamma^{1} \alpha \beta+\gamma^{1} a \delta+\gamma^{1} \beta \delta \\
& +\delta^{1} \alpha \beta+\delta^{1} \alpha \gamma+\delta^{1} \beta \gamma \\
& =3(\alpha \beta \gamma+\alpha \beta \delta+\alpha \gamma \delta+\beta \gamma \delta) \text {, } \\
& \mathrm{\nu}^{0}{ }^{0} \beta \gamma \equiv \alpha^{0} \beta \gamma+a^{0} \beta \delta+\alpha^{0} \gamma \delta+\beta^{0} a \gamma+\ldots \ldots \\
& =2(\beta \gamma+\beta \delta+\gamma \delta+\alpha \beta+\alpha \gamma+\alpha \delta)
\end{aligned}
$$

and $\Sigma \alpha^{0}=\alpha^{0}+\beta^{0}+\gamma^{0}+\delta^{0}=4$.

In the identity (5) read in this way, let us substitute for $p$ the values $m, m-1, m-2, \ldots m-r+1$, successively, multiply these equations by +1 and by -1 alternately, and add the results together. Noting that each term is of dimensions $m$, we have

$$
\begin{aligned}
& \mathrm{S}^{m}-(-1)^{r} \Sigma\left(a^{m-r} \beta \gamma \ldots\right) \\
& =\Sigma_{\alpha} \Sigma_{a^{m-1}}-\Sigma_{\alpha} \beta \Sigma_{a^{m-2}}+\Sigma_{\alpha} \beta \gamma \Sigma_{\alpha^{m-3}}-\ldots-(-)^{r} \Sigma(\alpha \beta \ldots) \Sigma^{m-r} .
\end{aligned}
$$

Hence we have

$$
\begin{aligned}
& \Sigma^{m}-\Sigma_{\alpha} \Sigma^{m-1}+\Sigma \alpha \beta \Sigma \alpha^{m-2}-\ldots+(-1)^{r} \Sigma(\alpha \beta \ldots) \Sigma a^{m-r} \\
& =(-1)^{r}\left(a^{m-r} \beta \gamma \ldots\right) \text {. }
\end{aligned}
$$

A further generalisation is got by subtracting this from the corresponding formula with $s$ substituted for $r$.

This gives

$$
\begin{aligned}
p_{r+1} s_{m-r-1}-p_{r+2} & s_{m-r-2}+p_{r+3} s_{m-r-3}-\ldots-(-1)^{s-r} p_{s} s_{m-s} \\
& =\triangle\left(a^{m-r} \beta \gamma \ldots\right)-(-1)^{s-r}-\left(a^{m-s} \beta \gamma \ldots\right) . .
\end{aligned}
$$

Now note the special case when $s=r+2$. Equation (9) then becomes

$$
p_{r+1} s_{m-r-1}-p_{r+2} s_{m-r-2}=\Sigma\left(a^{m-r} \beta \gamma \ldots\right)-\Sigma\left(u^{m-r-1} \beta \gamma \ldots\right) \text {. }
$$

This equation $(10)$ could also be easily deduced directly from first principles. 